\title{
Src homology phosphotyrosyl phosphatase 2 mediates cisplatin-related drug resistance by inhibiting apoptosis and activating the Ras/PI3K/Akt1/survivin pathway in lung cancer cells
}

\author{
CHUNLAN TANG, HU LUO, DAN LUO, HEPING YANG and XIANGDONG ZHOU \\ Department of Respiratory Medicine, The First Affiliated Hospital of The Third Military Medical University, \\ Shapingba, Chongqing 400038, P.R. China
}

Received February 17, 2017; Accepted October 20, 2017

DOI: 10.3892/or.2017.6109

\begin{abstract}
Cisplatin resistance is a major cause of chemotherapeutic failure in lung cancer patients. Unraveling the molecular mechanisms underlying cisplatin (CDDP) resistance is important in lung cancer therapeutics. To explore the role of Src homology phosphotyrosyl phosphatase 2 (SHP2) in the development of cisplatin resistance in lung cancer and the underlying mechanism, we established stable SHP2-overexpressing H446-SHP2-OE cells and SHP2-knockdown H446/CDDP-SHP2-shRNA cells derived from H446 and H446/CDDP (cisplatin-resistant) parental lung cancer cells. The cell viability and apoptosis of these cells exposed to CDDP were observed to determine the influence of SHP2 on drug resistance. In addition, the expression of SHP2, Ras, Akt1 and survivin was assessed by western blot analysis after the lung cancer cells were challenged by cisplatin or silenced by Ras siRNA. As a result, the 50\% inhibitory concentration $\left(\mathrm{IC}_{50}\right)$ of the H446-SHP2-OE cells exposed to CDDP increased from 1.01 to $1.218 \mu \mathrm{g} / \mathrm{ml}$ vs. the H446-control vector cells. The percentage of apoptotic cells was smaller in the H446-SHP2-OE cells vs. the H446-control vector cells after cisplatin challenge. In addition, the expression of Ras, pAkt1, Akt1 and survivin in the H446/CDDP cells was significantly increased vs. the H446 cells. Furthermore, the $\mathrm{IC}_{50}$ of the H446/CDDP-SHP2-shRNA cells exposed to CDDP decreased from 11.92 to $4.382 \mu \mathrm{g} / \mathrm{ml}$ vs. the H446/CDDP-mock cells. There were significantly more apoptotic cells among the H446/CDDP-SHP2-shRNA cells vs. the H446/CDDPmock cells exposed to cisplatin. A smaller percentage of the
\end{abstract}

Correspondence to: Professor Xiangdong Zhou, Department of Respiratory Medicine, First Affiliated Hospital of the Third Military Medical University, 30 Gao Tanyan Street, Shapingba, Chongqing 400038, P.R. China

E-mail: 3375869541@qq.com

Key words: SHP2, lung cancer, cisplatin resistance, apoptosis, Ras/PI3K/Akt1/survivin
H446/CDDP-SHP2-shRNA cells vs. the H446/CDDP-mock cells was observed. In addition, the expression of pAktl and survivin in the H446, H446/CDDP and H446/CDDP-mock cells was increased upon exposure to cisplatin however, a corresponding change was not observed in the H446/CDDPSHP2-shRNA cells. Upon Ras RNA silencing with cisplatin, the Ras expression was significantly decreased in the H446, H446-SHP2-OE and H446/CDDP cells. However, upon Ras RNA interference, the SHP2 expression was not significantly changed, but the expression of Akt1, pAkt1 and survivin was significantly increased in the H446-SHP2-OE and H446/CDDP cells. In conclusion, SHP2 is a new cisplatin resistance-related phosphatase in lung cancer, which inhibits apoptosis by activating the Ras/PI3K/Akt1/survivin signaling pathway.

\section{Introduction}

Lung cancer is the most common cancer and a leading cause of cancer-associated mortality worldwide (1-3). Non-small cell lung cancer (NSCLC) is the most common type of lung cancer and is associated with high mortality and poor prognosis; over $70 \%$ of the patients have advanced cancer at the time of diagnosis and are not suitable for surgical treatment $(4,5)$. Therefore, chemotherapy is the main therapeutic option available for these patients $(6,7)$. Two platinum-containing chemotherapy regimens are regularly used as the first-line treatment for patients with lung cancer $(8,9)$. However, drug resistance is a major challenge with this line of treatment $(10,11)$. Intrinsic changes in tumor cells are known to play a critical role in the emergence of chemoresistance (12-14). Thus, the identification of the associated mechanisms is essential in cancer therapeutics.

Cisplatin is the most active platinum agent for patients with advanced lung cancer and early-stage patients who require adjuvant therapy (15). However, after treatment for a certain period of time, most patients develop cisplatin resistance $(16,17)$.

Inhibiting the apoptosis of cancer cells is one mechanism of cisplatin resistance (18). Survivin is an apoptosis protein-inhibitor that is upregulated in cancer cells and serves as an attractive prognostic marker of malignancies (19).

Src homology 2 (SH2) domain containing protein tyrosine phosphatase-2 (SHP2) promotes signaling for most growth 
factors and cytokine receptors. SHP2 is required for growth factor/cytokine-induced cell proliferation, migration and survival in many cancers (20) and is essentially mediated via activating the Ras/mitogen-activated protein kinase (MAP) pathway (7,21-23). However, the influence of SHP2 in inducing lung cancer cell resistance to cisplatin is not well established.

The phosphoinositol 3-kinase (PI3K/Akt) pathway is involved in cell survival, differentiation, proliferation, apoptosis and metastasis and is implicated in the pathogenesis of many tumors (24).

The Ras/MAPK pathway has been the preferential pathway used in cancer research $(25,26)$. SHP2 is a direct activator of Ras and the downstream of MAPK signaling, which are involved in tumor progression (27).

We previously discovered upregulated SHP2 in lung cancer tissues. Inhibiting SHP2 downregulated the expression of Akt (28). Furthermore, inhibiting the PI3K/Akt pathway reversed resistance of the A549/CDDP cells to cisplatin, while activation of the PI3K/Akt pathway enhanced tolerance of the A549/CDDP cells to cisplatin. However, whether the anti-apoptotic effect of the Ras-activated PI3K/Akt pathway, is also involved in the SHP2-enhanced lung cancer resistance to cisplatin is not known.

The aim of this study was to explore the role of SHP2 in the development of cisplatin resistance in lung cancer as well as the underlying mechanism.

\section{Materials and methods}

Statement of ethics. The experimental protocol was approved by the Human Ethics Committee of the First Affiliated Hospital of the Third Military Medical University.

Cell lines andreagents. The human small cell lung cancer (SCLC) H446 and adenocarcinoma SPC-A-1 cell lines were obtained from the Shanghai Institute of Biochemistry and Cell Biology (Shanghai, China). The H446/CDDP and SPC-A-1/CDDP cells were sourced from Dr Linzhi Liu at Xinqiao Hospital of the Third Military Medical University. Cisplatin was obtained from North China Pharmaceutical Co., Ltd. (Shijiazhuang, China). The Cell Counting Kit-8 (CCK-8) was obtained from Dojindo Molecular Technologies, Inc. (Kumamoto, Japan) and the H-Ras siRNA and antibodies from Santa Cruz Biotechnology, Inc. (Santa Cruz, CA, USA). The bicinchoninic acid (BCA) protein assay kit and the enhanced chemiluminescent detection kit were purchased from Pierce Biotechnology, Inc. (Rockford, IL, USA). The RNAiso reagent was obtained from Takara Bio, Inc. (Shiga, Japan). The cDNA reverse transcription kit was purchased from Fermentas Inc. (Burlington, ON, Canada). The FITC Annexin V and PI apoptosis detection kit was obtained from BD Biosciences (San Diego, CA, USA).

Cell culture and treatment. The cells were cultured in RPMI-1640 medium containing $10 \% \mathrm{FBS}$ at $37^{\circ} \mathrm{C}$ with $5 \% \mathrm{CO}_{2}$ and $100 \%$ humidity and treated with different concentrations of cisplatin for predefined time-points.

Immunohistochemistry. The cells were seeded over cover slips, fixed with $4 \%$ paraformaldehyde, permeabilised with $1 \%$ Triton X-100, blocked with $3 \% \mathrm{H}_{2} \mathrm{O}_{2}$ and then incubated with primary antibody after being blocked in $0.5 \%$ normal goat serum. The immunoreactivity of the cells was assessed by staining intensity and scored on a scale of 0 to 3 as follows: 0 (-), absence of staining; 1 (+), weak staining; $2(++)$, moderate staining; and $3(+++)$, strongly immunoreacting. The cells incubated with primary antibody diluents without the primary antibody served as a negative control.

SHP2 enzyme activity assay. Protein (50 mg) was mixed with protein tyrosine phosphatase (PTP) buffer $(25 \mathrm{mM}$; $\mathrm{pH}$ 6.0 Tris- $\mathrm{HCl}, 50 \mathrm{mM} \mathrm{NaCl}, 2 \mathrm{mM}$ EDTA and $10 \mathrm{mM}$ DTT) to a final volume of $99 \mu \mathrm{l}$. Subsequently, $1 \mu \mathrm{l}$ of $1 \mathrm{M}$ para-nitrophenyl phosphate (pNPP) was added and incubated at $37^{\circ} \mathrm{C}$ for $10 \mathrm{~min}$, and then terminated with $900 \mu \mathrm{l}$ of termination buffer $(1 \mathrm{ml}$ of $5 \mathrm{~N} \mathrm{NaOH}$ plus $5 \mathrm{ml}$ of $50 \%$ alcohol $+4 \mathrm{ml}$ of Milli-Q $\mathrm{H}_{2} \mathrm{O}$ ). The optical density (OD) was determined at $405 \mathrm{~nm}$. A PTP buffer $(99 \mu \mathrm{l})$ without protein was used as a negative control.

Construction of SHP2 lentiviral vectors and cell transfection. To construct an SHP2 overexpression lentiviral vector, we designed and synthesized the primers engineered with the BamH1 restriction site in the forward direction and the Xhol restriction site in the reverse direction, according to the full-length sequence of SHP2 in PubMed database (NM_002834) and the restriction enzyme cutting sites of the lentiviral vector. cDNA from the H446 cells was amplified and cloned into the PTA2 vector, and then transfected into competent Escherichia coli (DH5 $\alpha$ ) cells. The SHP2 overexpression lentiviral vector LV-SHP2-IRES-PURO was constructed using conventional methods. An empty LV-IRESPURO vector was used as a negative control.

The knockdown of the SHP2 gene was also performed using lentivirus-mediated RNA interference. Briefly, oligonucleotides coding for the short hairpin RNA (shRNA) targeting human SHP2 were annealed and inserted into the pFIV-GFP-PURO vector to produce the lentiviral expression vector pFIV-GFP-PURO-shSHP2. The integrity was confirmed on sequencing. An empty pFIV-GFP-PURO vector was used as a negative control.

The SHP2 overexpression vector or SHP2 shRNA vector and empty control vectors, together with the corresponding packaged plasmids were transfected into the HEK293T cells to generate lentiviral particles carrying target sequences. Virus supernatants were collected $72 \mathrm{~h}$ post-transfection.

The H446 cells were infected with lentiviral particles containing the SHP2 overexpression sequence to produce stable H446-SHP2-OE and H446-control vector target cell lines after puromycin selection $(2 \mu \mathrm{g} / \mathrm{ml})$. The H446/CDDP cells were infected with lentiviral particles containing the SHP2 shRNA sequence to produce stable H446/CDDPSHP2-shRNA and H446/CDDP-mock target cell lines after puromycin selection $(2 \mu \mathrm{g} / \mathrm{ml})$. The efficiency of overexpression or knockdown of SHP2 in the H446-SHP2-OE and the H446/CDDP-SHP2-shRNA cells respectively, was assessed by both RT-PCR and western blot analysis.

Ras siRNA transfection. Three eppendorf tubes (A, B and C) were prepared with $500 \mu \mathrm{l}$ of RPMI-1640 medium in each. H-Ras siRNA $(24 \mu \mathrm{l})$ was added in tube A and $12 \mu \mathrm{l}$ of 


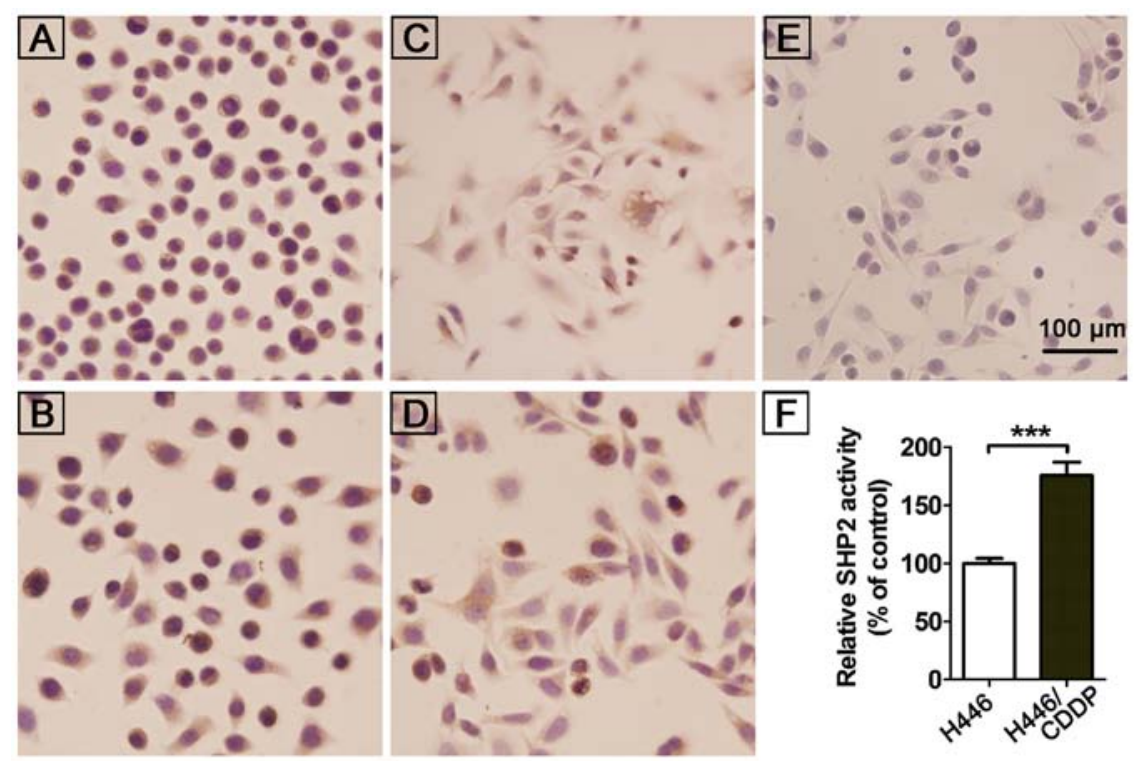

Figure 1. SHP2 expression and enzyme activity in cisplatin-resistant cells and parental cells. The SHP2 expression was determined by immunohistochemistry in (A) SPC-A-1, (B) SPC-A-1/CDDP, (C) H446, (D) H446/CDDP and (E) negative control, H446 cells incubated with primary antibody diluent without primary antibody (magnification, x400). (F) Relative SHP2 activity in H446 and H446/CDDP cells. Representative data shown from one of the three independent experiments with similar results. ${ }^{* * *} \mathrm{P}<0.001$ vs. the control. SHP2, Src homology phosphotyrosyl phosphatase 2; CDDP, cisplatin.

Lipofectamine 2000 reagent was added in tubes B and $\mathrm{C}$, at room temperature (RT) for 5 min. Tubes A and B were mixed and cultured at RT for $20 \mathrm{~min}$. A $500 \mu \mathrm{l}$ mixture of A and $\mathrm{B}$ was added to cells in two culture bottles with $60 \%$ confluency and $1.5 \mathrm{ml}$ fresh medium. A $500 \mu 1$ of solution in C tube was added to another bottle with cells and incubated for $8 \mathrm{~h}$ after which $5 \mathrm{ml}$ of complete growth medium without antibiotics was added. The transfection mixture was removed and incubated for an additional $40 \mathrm{~h}$. The efficiency of Ras RNA interference was assessed by both gel based RT-PCR and western blot analysis.

Cell viability assay. Cell viability was detected using the Cell Counting Kit-8 (CCK-8) according to the manufacturer's instructions. $\mathrm{The} \mathrm{IC}_{50}$ of cisplatin was calculated.

Flow cytometric analysis. An FITC Annexin V and PI apoptosis detection kit was used to evaluate apoptosis, according to the manufacturer's protocol.

Western blot analysis. Equal quantities of total protein were run on SDS-PAGE and transferred onto PVDF membranes, blocked with 5\% nonfat milk, incubated with primary antibodies, incubated with peroxidase-conjugated secondary antibodies and developed with enhanced chemiluminescent detection reagent.

RNA extraction and gel based real-time PCR analysis. Total RNA was isolated and cDNA was synthesized routinely. SYBR Green I was used for RT-PCR analysis. The RT-PCR products were run on the agarose gel and the quantitative gel images were obtained.

Statistical analysis. Results are presented as the mean \pm standard deviation (SD). Statistical analysis was performed using SPSS 18.0 software (SPSS Inc., Chicago, IL, USA).
Between-group differences were assessed using the Student's t-test and one-way analysis of variance (ANOVA) was used for multi-group comparisons. $\mathrm{P}<0.05$ was considered to indicate a statistically significant difference.

\section{Results}

Expression and enzyme activity of SHP2 in cisplatin-resistant human lung cancer cells. Our previous study found a significantly higher expression of SHP2 in NSCLC by tissue microarray and also in NSCLC patients with lymph node metastasis vs. patients without lymph node metastasis (29). The results were consistent with previous in vivo observations by immunohistochemical detection of SHP2 expression in cisplatin-induced drug resistant cells (H446/ CDDP, SPC-A-1/CDDP) and their parental cell lines (H446, SPC-A-1). A high expression of SHP2 (+++) was observed in the cytoplasm of all tested cells (Fig. 1A-E). There was no significant difference in the SHP2 expression between the cisplatin-resistant and the parental cells. However, further investigation revealed a significantly higher activity of SHP2 in cisplatin-resistant H446/CDDP cells vs. H446/CDDP cells (Fig. 1F). This indicates an involvement of SHP2 in cisplatin resistance of lung cancer cells.

Validation of constructed recombinant human lung cancer cells. To further investigate whether SHP2 is required for the development of cisplatin resistance in lung cancer cells, we separately induced overexpression and knockdown of the SHP2 expression in H446 and H446/CDDP cells. As illustrated in Fig. 2A-C, SHP2 expression in the H446-SHP2-OE cells was significantly higher than that in the H446 and H446-control vector cells. In addition, SHP2 expression in the H446/CDDP-SHP2-shRNA cells was significantly lower than that in the H446/CDDP and H446/CDDP-mock cells. These 
A

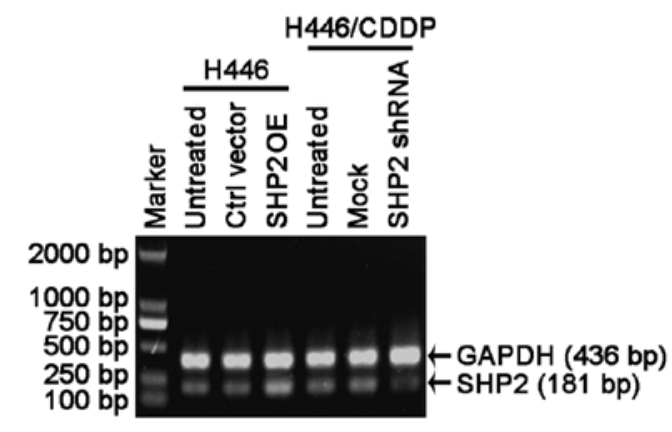

B

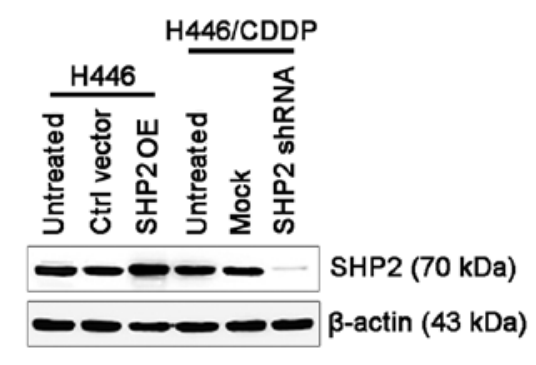

C

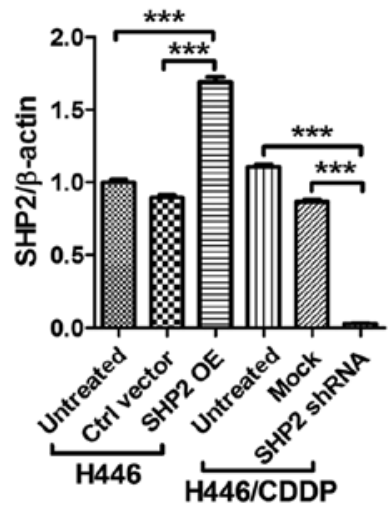

Figure 2. Efficiency of the overexpression or knockdown of SHP2 in H446-SHP2-OE and H446/CDDP-SHP2-shRNA cells as assessed by (A) RT-PCR and (B and C) western blot analysis. (B) The bands of the western blot analysis. (C) The quantification of the relative expression level of each test band of the western blot analysis. Cells from left to right: untreated H446, H446-control vector, H446-SHP2-OE, untreated H446/CDDP, H446/CDDP-mock, H446/ CDDP-SHP2-shRNA. ${ }^{* * * *}$ p $<0.001$. CDDP, cisplatin; SHP2, Src homology phosphotyrosyl phosphatase 2; OE, overexpression.
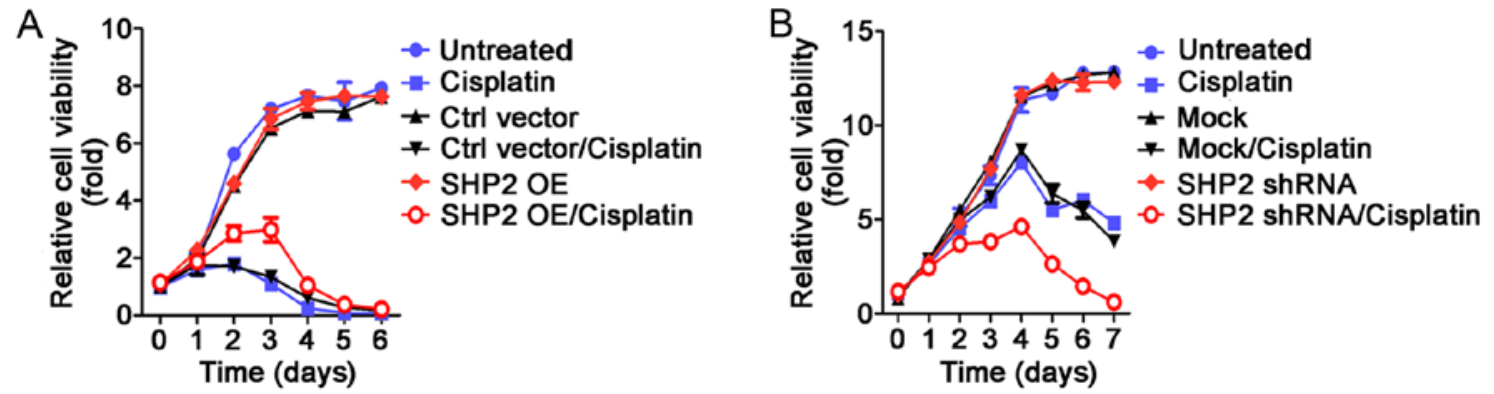

Figure 3. Relative viability of lung cancer cells in response to cisplatin treatment. (A) The relative cell viability of H446, H446-control vector and H446-SHP2-OE cells was assessed by CCK-8 assay in the presence or absence of cisplatin $(1 \mu \mathrm{g} / \mathrm{ml})$ for $24 \mathrm{~h}$. (B) The relative cell viability of H446/CDDP, H446/CDDP-mock and H446/CDDP-SHP2-shRNA cells was assessed by CCK-8 assay in the presence or absence of cisplatin (1 $\mu \mathrm{g} / \mathrm{ml})$ for $24 \mathrm{~h}$. Representative data shown from one of three independent experiments with similar results. CDDP, cisplatin; SHP2, Src homology phosphotyrosyl phosphatase 2; OE, overexpression.

results confirmed the successful generation of transgenic cells with the overexpression or knockdown of SHP2.

SHP2 protects lung cancer cells exposed to cisplatin. To investigate the possible involvement of SHP2 in cisplatininduced drug resistance in lung cancer patients, cells challenged with cisplatin were subjected to CCK- 8 assay. The results revealed no significant difference in the relative cell viability of the H446, H446-control vector and the H446SHP2-OE cells without cisplatin (Fig. 3A), which indicated that the inserted vectors had no influence on the H446 cell viability. However, the relative cell viability of the three cell lines was significantly inhibited with cisplatin $(1 \mu \mathrm{g} / \mathrm{ml})$ vs. the same type of cells without cisplatin $(\mathrm{p}<0.05)$. In addition, the relative cell viability of the H446-SHP2-OE cells was significantly increased than that of the other two cell lines $(\mathrm{p}<0.05)$. This indicated that upregulation of SHP2 significantly decreased the sensitivity of the $\mathrm{H} 446$ cells to cisplatin.

The influence of SHP2 knockdown on the sensitivity of human lung cancer cells to cisplatin was further explored. The H446/CDDP, H446/CDDP-mock and H446/CDDP-SHP2-shRNA cells exhibited similar relative cell viability following treatment with cisplatin $(1 \mu \mathrm{g} / \mathrm{ml})$ (Fig. 3B), which excluded a possible influence of the inserted vector on cell viability. However, the relative cell viability of the three cell lines was significantly lower following treatment with cisplatin $(1 \mu \mathrm{g} / \mathrm{ml})$ vs. that of the controls without cisplatin $(\mathrm{p}<0.05)$. The relative cell viability of the H446/CDDP-SHP2-shRNA cells following treatment with cisplatin was significantly lower vs. that of the other two cell lines $(\mathrm{p}<0.05)$. This indicated that sensitivity of the H446/CDDP cells to cisplatin was increased by downregulating SHP2.

To quantitatively assess the influence of SHP2 on lung cancer cell sensitivity to cisplatin, the $\mathrm{IC}_{50}$ for each cell line was calculated. The H446-control vector and the H446-SHP2-OE cells were treated with cisplatin $(0.05,0.25$, $1.25,5$ and $10 \mu \mathrm{g} / \mathrm{ml}$ ), and the H446/CDDP-mock and H446/ CDDP-SHP2-shRNA cells were treated with cisplatin $(5,10$, 20,40 and $80 \mu \mathrm{g} / \mathrm{ml}$ ) for $24 \mathrm{~h}$. The $\mathrm{IC}_{50}$ of the H446-control vector, the H446-SHP2-OE, the H446/CDDP-mock and the H446/CDDP-SHP2-shRNA cells to cisplatin was 1.01, 1.218, 11.92 and $4.382 \mu \mathrm{g} / \mathrm{ml}$, respectively. Collectively, these results confirmed the enhanced tolerance of $\mathrm{H} 446$ cells to cisplatin after upregulating SHP2, while downregulating SHP2 increased the sensitivity of $\mathrm{H} 446$ cells to cisplatin.

SHP2 induces drug resistance via inhibition of cisplatin-induced apoptosis in lung cancer cells. To investigate whether SHP2-induced chemoresistance was mediated by antiapoptosis, flow cytometric analysis was performed to assess apoptosis of the H446-control vector, the H446-SHP2-OE, 

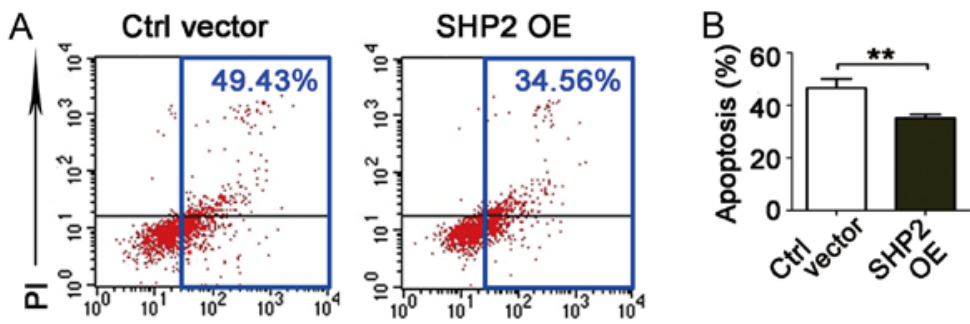

Annexin V
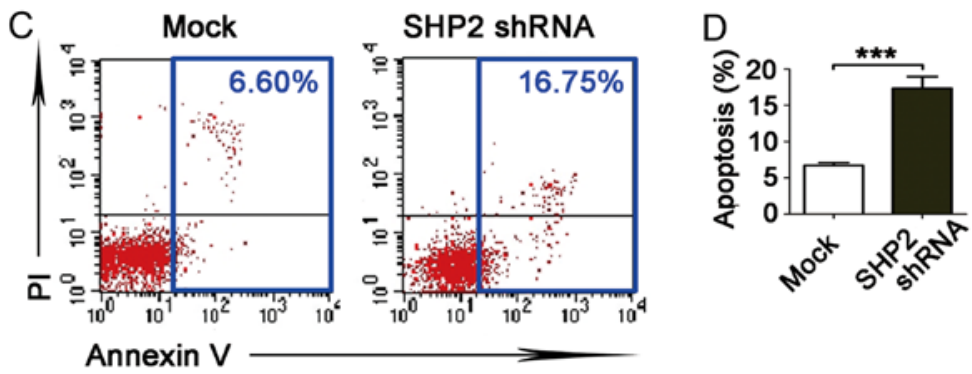

Figure 4. SHP2 inhibits apoptosis in cisplatin-resistant lung cancer cells. Flow cytometric analysis was performed to assess apoptosis (A and B) of the H446-control vector and the H446-SHP2-OE cells and (C and D) the H446/CDDP-mock and H446/CDDP-SHP2-shRNA cells in response to cisplatin $(1.0 \mu \mathrm{g} / \mathrm{ml})$ treatment for $24 \mathrm{~h}$. Panels A and C show representative data from three iterations of flow cytometric analysis; the average percentage of apoptosis is presented in panels $\mathrm{B}$ and $\mathrm{D}$, where the percentage of early apoptotic (Annexin $\mathrm{V}^{+} / \mathrm{PI}$ ) and late apoptotic (Annexin $\mathrm{V}^{+} / \mathrm{PI}^{-}$) cells were combined for analysis ${ }^{* *} \mathrm{P}<0.05,{ }^{* * * *} \mathrm{P}<0.05$. CDDP, cisplatin; SHP2, Src homology phosphotyrosyl phosphatase 2.

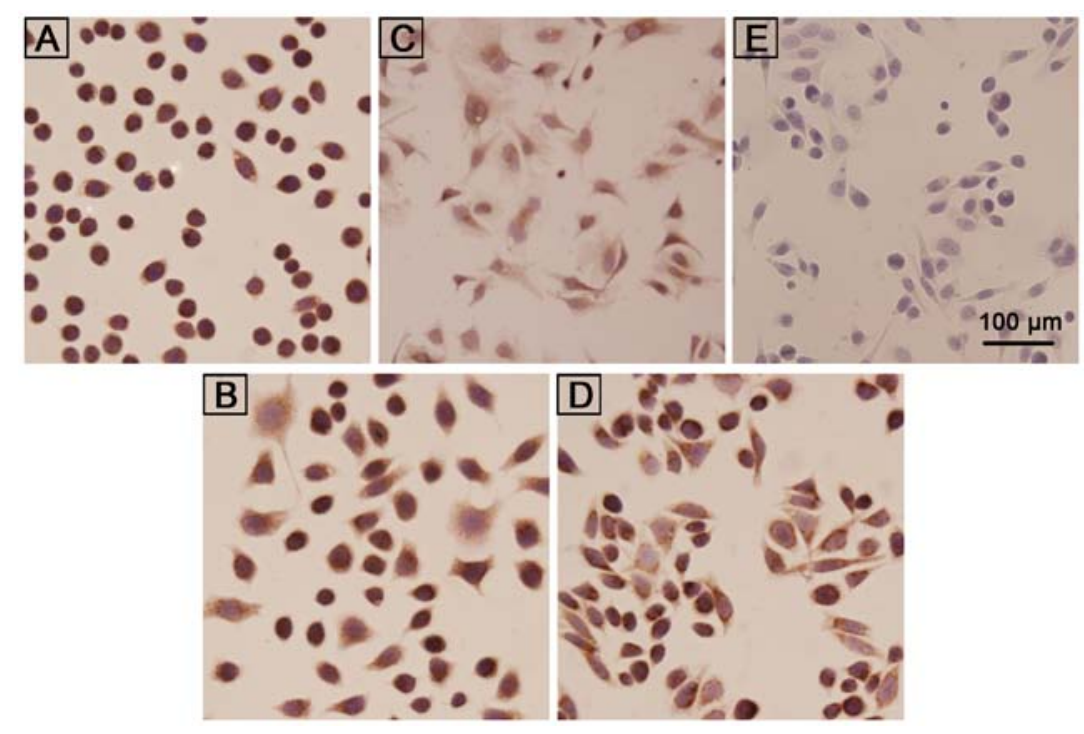

Figure 5. Ras expression in cisplatin-induced drug-resistant and parental cells. The Ras expression was determined by immunohistochemistry in (A) SPC-A-1, (B) SPC-A-1/CDDP, (C) H446, (D) H446/CDDP and (E) negative control, H446 cells incubated with primary antibody diluents without primary antibody (magnification, $\mathrm{x} 400)$. CDDP, cisplatin.

H446/CDDP-mock and the H446/CDDP-SHP2-shRNA cells in response to cisplatin $(1.0 \mu \mathrm{g} / \mathrm{ml})$.

The results revealed that SHP2 overexpression significantly decreased lung cancer cell apoptosis and apoptosis due to cisplatin was significantly lower in the H446-SHP2-OE vs. the H446-control vector cells (average of 35.15 vs. $46.64 \%$; Fig. 4A and B). In contrast, SHP2 knockdown significantly increased apoptosis. The apoptosis rate due to cisplatin was significantly higher in the H446/CDDP-SHP2-shRNA vs. the H446/CDDP-mock cells (average of 17.35 vs. $6.73 \%$; Fig. 4C and D). These observations indicated that upregulation of SHP2 protected lung cancer cells from apoptosis and enhanced their resistance to cisplatin.

SHP2 Ras/PI3K/Akt/survivin pathway is critical to chemotherapeutic outcomes in lung cancer. To investigate whether the Ras-activated PI3K/Akt pathway, downstream of MAPK, plays a critical role in inhibiting apoptosis and is involved in SHP2-induced lung cancer cell resistance to cisplatin, the Ras expression in lung cancer cells was assessed by immunohistochemistry. A high Ras expression (+++) was observed in both SPC-A-1 and cisplatin-induced drug resistant 


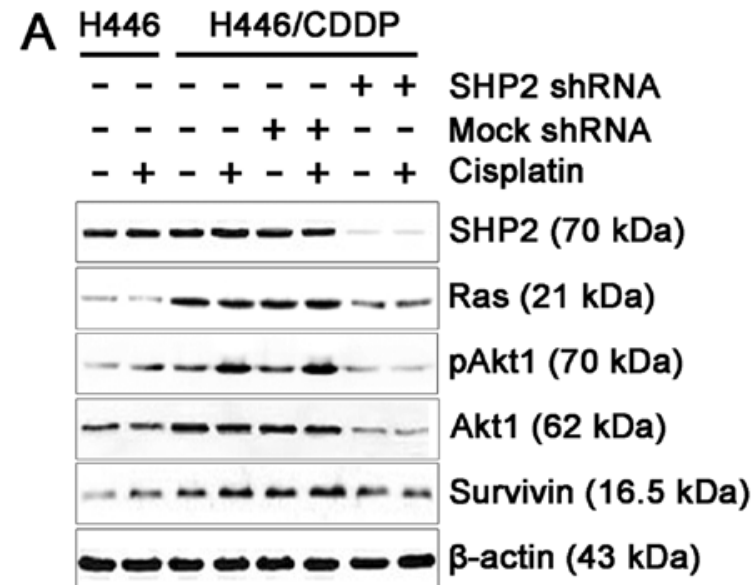


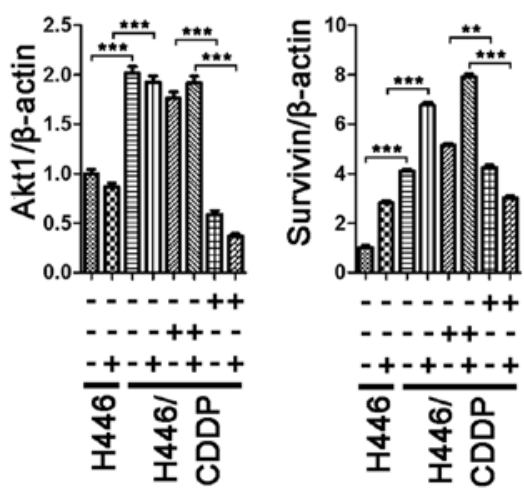

C
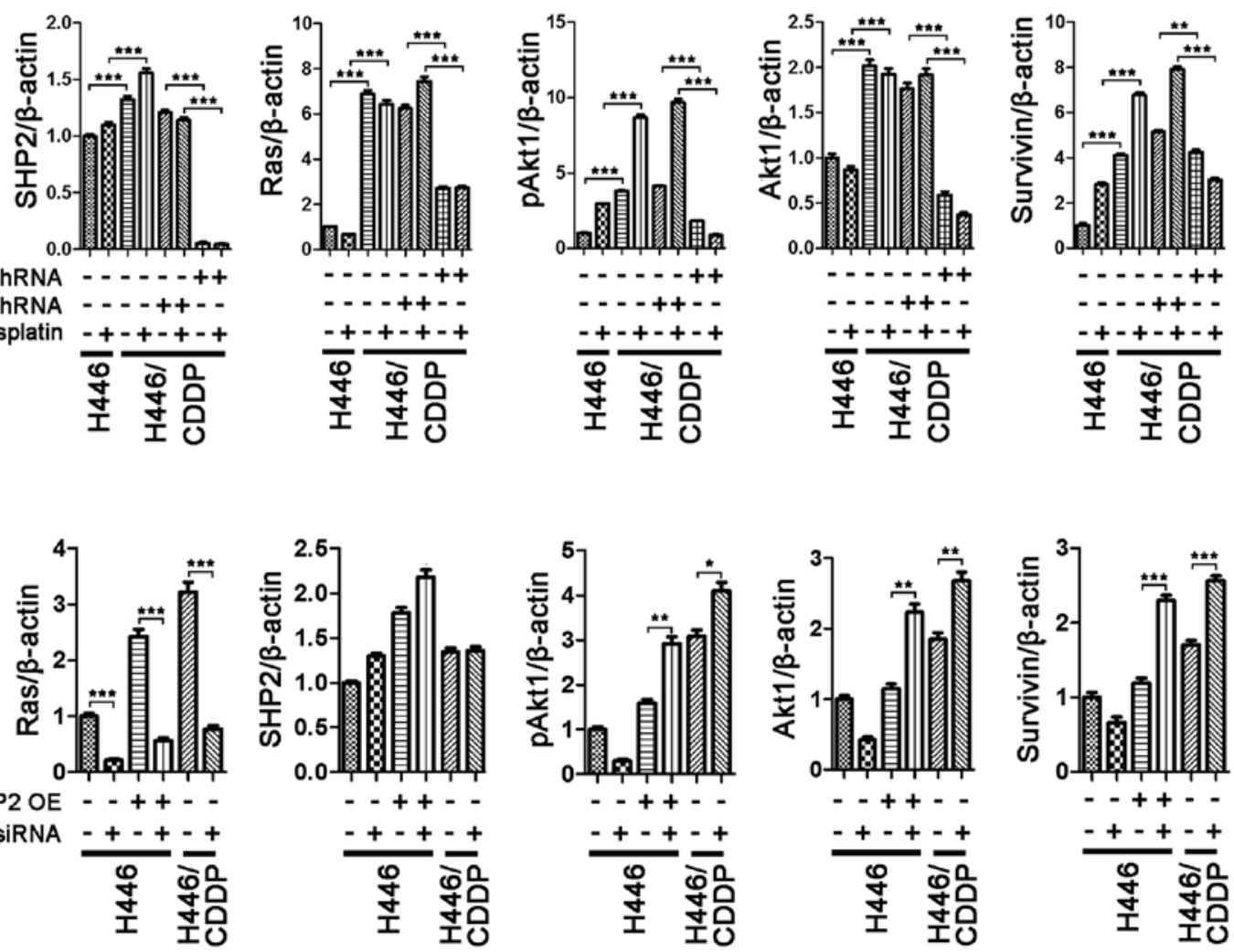

Figure 6. Ras-mediated PI3K/Akt pathway is activated by SHP2 in cisplatin-resistant lung cancer cells. (A and C) The expression of SHP2, Ras, Akt1, pAkt1 and survivin in the H446/CDDP, H446/CDDP-mock and H446/CDDP-SHP2-shRNA cells treated with cisplatin (1.0 $\mu \mathrm{g} / \mathrm{ml})$ for $24 \mathrm{~h}$ assessed by western blot analysis. In (C) the bars in the histogram represent, from left to right, the following cells: H446, H446 + CDDP, H446/CDDP, H446/CDDP + CDDP, H446/CDDPmock, H446/CDDP-mock + CDDP, H446/CDDP-SHP2-shRNA and H446/CDDP-SHP2-shRNA+ CDDP cells. (B and D) The expression of SHP2, Ras, Akt1, pAkt1 and survivin in H446, H446-SHP2-OE and H446/CDDP cells before and after Ras RNA interference assessed by western blot analysis; In (B) the bars in the histogram represent, from left to right, the following cells: H446, H446-Ras siRNA, H446-SHP2-OE, H446-SHP2-OE-Ras siRNA, H446/CDDP and H446/ CDDP-Ras siRNA cells. (C) shows quantification of the relative expression level of each test band in (A). (D) shows quantification of the relative expression level of each test band in (B). ${ }^{*} \mathrm{p}<0.05,{ }^{* *} \mathrm{p}<0.01,{ }^{* * * *} \mathrm{p}<0.001$. CDDP, cisplatin; SHP2, Src homology phosphotyrosyl phosphatase 2; OE, overexpression.

SPC-A-1/CDDP cells (Fig. 5A and B). The expression of Ras in cisplatin-induced drug resistant H446/CDDP cells (+++) was greater than that in the $\mathrm{H} 446$ cells $(++)$ (Fig. 5C and D).

To gain an insight into the molecular mechanisms by which Ras further promoted lung cancer cell resistance to cisplatin, H446, H446/CDDP, H446/CDDP-mock and H446/CDDP-SHP2-shRNA cells were treated with cisplatin $(1.0 \mu \mathrm{g} / \mathrm{ml})$ for $24 \mathrm{~h}$. The changes in expression of SHP2, Ras, pAkt1, Akt1 and survivin were assessed by western blot analysis. The expression of Ras, pAkt1, Akt1 and survivin in the H446/CDDP cells was significantly higher vs. that in the H446 cells without cisplatin resistance, which indicated that these proteins are closely associated with cisplatin resistance (Fig. 6A and $\mathrm{C}$ ). Furthermore, the expression of Ras, Akt1, pAkt1 and survivin in H446/CDDP-SHP2shRNA cells was lower than that in the H446/CDDP-mock cells, which is consistent with the SHP2 expression. This indicated that SHP2 may be an upstream regulatory factor 
of Ras, Akt1 and survivin, which may directly upregulate the PI3K/Akt1 pathway. The expression of pAkt1 and survivin was also increased following exposure of these cells to cisplatin. The SHP2 expression was not inhibited in the H446, H446/CDDP and H446/CDDP-mock cells, which confirmed the involvement of these two factors and the antiapoptotic effect. However, no significant differences were observed in the expression of these two proteins, before or after cisplatin exposure, in the H446/CDDP-SHP2-shRNA cells after the SHP2 knockdown. This indicates that cisplatin activated SHP2 which in turn downregulated apoptosis via its effect on the SHP2/Ras/PI3K/Akt1/survivin pathway. This further confirmed that SHP2 participated in pAkt1- and survivin-induced cisplatin resistance in lung cancer cells.

Upon Ras RNA silencing with cisplatin, the expression of Ras was significantly decreased in the H446, H446-SHP2-OE and the H446/CDDP cells. However, the SHP2 expression was not significantly changed following Ras RNA interference (Fig. 6B and D). The expression of Akt1, pAkt1 and survivin was significantly increased in the H446-SHP2-OE and H446/CDDP cells after the Ras RNA interference. These results revealed that Ras was not the only mediator of SHP2induced apoptosis inhibition, via the PI3K/Akt1 pathway, in inducing cisplatin resistance. There may be other compensatory ways to increase the expression of Akt1 and pAkt1. Collectively, we demonstrated that SHP2 was a direct activator of Ras. Inhibiting the Ras expression regulated the PI3K/Akt pathway. Therefore, the SHP2/Ras/PI3K/Akt/survivin pathway could be a potential therapeutic target for cisplatin-related drug resistance in lung cancer patients.

\section{Discussion}

Lung cancer is a highly malignant neoplasm. About $75 \%$ of all patients are diagnosed at an advanced stage and have lost the opportunity for surgery, thus chemotherapy is often the only viable option. Chemotherapy can considerably increase survival, alleviate symptoms and improve the quality of life of lung cancer patients. However, the development of chemoresistance is a major cause of chemotherapy failure. Thus, combination therapy directed to an appropriate target may improve therapeutic efficacy (30).

We previously reported a positive expression of SHP2 in NSCLC tissues, but not in adjacent and normal lung tissues, which indicated the importance of SHP2 in NSCLC (31). Indeed, SHP2 is considered as a potential marker and therapeutic target for NSCLC and SHP2 inhibition may play a role in combination therapeutics. However, the involvement of SHP2 in lung cancer resistance has not been reported in the literature.

In the present study, cisplatin-induced drug-resistant cells SPC-A-1/CDDP and H446/CDDP were generated from SPC-A-1 and H446 cells, respectively (30). A high expression of SHP2 was observed in these cells. We found significantly higher Ras expression in the H446/CDDP cells as compared to that in the $\mathrm{H} 446$ cells, while no significant difference was observed between the SPC-A-1/CDDP and the SPC-A-1 cells. This may be due to the fact that lung adenocarcinoma typically exhibits primary resistance, while SCLC exhibits acquired resistance to cisplatin treatment. Our results support the hypothesis that SHP2 is associated with cisplatin-induced drug resistance in lung cancer.

Drug sensitivity tests revealed that overexpression of SHP2 reduced the sensitivity of the $\mathrm{H} 446$ cells to cisplatin ( $\mathrm{IC}_{50}$ : 1.218 vs. $1.01 \mu \mathrm{g} / \mathrm{ml}$ ) and inhibition of SHP2 significantly reversed the tolerance capacity of the H446/CDDP cells to cisplatin $\left(\mathrm{IC}_{50}: 4.382\right.$ vs. $\left.11.92 \mu \mathrm{g} / \mathrm{ml}\right)$. Our findings indicated that SHP2 is a cisplatin resistance-associated protein in lung cancer. However, no significant differences were observed in SHP2 mRNA and the protein expression between H446 and H446/CDDP cells.

We further found significantly higher activity of SHP2 in H446/CDDP cells vs. H446 cells (OD value: $0.488 \pm 0.015$ vs. $0.249 \pm 0.011, \mathrm{P}<0.05)$, which indicated that enzymatic activity of SHP2 is involved in acquired resistance of H446 cells to cisplatin.

Overexpression or knockdown of SHP2 had no significant influence on the growth of H446 cells or H446/CDDP cells without cisplatin. However, cell proliferation was significantly inhibited with cisplatin. The lowest extent of inhibition was in cells with SHP2 overexpression while the greatest was in SHP2-knockdown cells. These results strongly indicated that increased SHP2 expression enhanced the tolerance of lung cancer cells to cisplatin. Inhibiting SHP2 reversed this tolerance and increased the sensitivity of lung cancer cells to cisplatin.

Inhibition of apoptosis is a mechanism of cisplatin resistance (14). After cisplatin treatment, we found that overexpression of SHP2 reduced apoptosis of the H446 cells (from 46.64 to $35.15 \%$ ), while inhibition of SHP2 increased apoptosis of the H446/CDDP cells (from 6.73 to $17.35 \%$ ). The expression of Ras, pAkt1, Akt1 and survivin was also significantly increased in the H446/CDDP cells vs. the H446 cells and decreased in the H446/CDDP-SHP2-shRNA cells vs. the H446/CDDP-mock cells, which was consistent with SHP2 expression. These results confirmed the contribution of SHP2 in the acquisition of cisplatin-induced drug resistance, which is mediated via the regulation of cell apoptosis which may be mediated via the PI3K/Akt pathway, an important anti-apoptotic pathway.

SHP2 is involved in cell growth, migration, invasion and transformation via several signaling pathways. Among these, the Ras/ERK and the PI3K/Akt pathways are considered to be closely associated with oncogenesis and tumor development (16). The present study revealed that inhibition of SHP2 was associated with reduced expression of Ras, Akt1, pAktl and survivin. However, no significant difference in SHP2 expression was found after silencing Ras in H446, H446-SHP2-OE and H446/CDDP cells exposed to cisplatin. These findings indicated that SHP2 is the upstream of Ras, Akt1 and survivin. SHP2 may be involved in drug resistance induced by cisplatin in lung cancer cells via the regulation of the Ras/PI3K/Akt1/survivin pathway. Interestingly, the expression of Akt1 and pAkt1 was decreased in the H446 cells, but increased in the H446-SHP2-OE and H446/CDDP cells after the Ras inhibition. We assumed that the SHP2 activation may increase the expression of both Akt1 and pAkt1 via other compensatory pathways independent of Ras, such as the JAK/STAT signaling pathway.

In conclusion, our findings revealed that SHP2 is associated with cisplatin-induced drug resistance in lung cancer 
and SHP2 directly activates Ras, which in turn regulates the $\mathrm{PI} 3 \mathrm{~K} / \mathrm{Akt}$ pathway. Only in vitro experiments were conducted in the present study, whereas further in vivo studies are currently ongoing by our research group.

\section{Acknowledgements}

This study was supported by grants from the National Nature Science Foundation (no. 81071913).

\section{References}

1. Torre LA, Siegel RL and Jemal A: Lung cancer statistics. Adv Exp Med Biol 893: 1-19, 2016.

2. Rivera MP: Lung cancer in women: Differences in epidemiology, biology, histology, and treatment outcomes. Semin Respir Crit Care Med 34: 792-801, 2013.

3. Mohan V, Agarwal R and Singh RP: A novel alkaloid, evodiamine causes nuclear localization of cytochrome-c and induces apoptosis independent of p53 in human lung cancer cells. Biochem Biophys Res Commun 477: 1065-1071, 2016.

4. D'Amico TA: Angiogenesis in non-small cell lung cancer. Semin Thorac Cardiovasc Surg 16: 13-18, 2004.

5. Müller-Tidow C, Diederichs S, Thomas M and Serve H: Genome-wide screening for prognosis-predicting genes in early-stage non-small-cell lung cancer. Lung Cancer 45 S145-S150, 2004.

6. Rich JN and Bao S: Chemotherapy and cancer stem cells. Cell Stem Cell 1: 353-355, 2007.

7. Dittrich PS and Manz A: Lab-on-a-chip: Microfluidics in drug discovery. Nat Rev Drug Discov 5: 210-218, 2006.

8. Sampsonas F, Ryan D, McPhillips D and Breen DP: Molecular testing and personalized treatment of lung cancer. Curr Mol Pharmacol 7: 22-32, 2014.

9. Dimou A and Papadimitrakopoulou V: Non-small cell lung cancer beyond biomarkers: The evolving landscape of clinical trial design. J Pers Med 4: 386-401, 2014.

10. Pass HI, Lavilla C, Canino C, Goparaju C, Preiss J, Noreen S, Blandino $\mathrm{G}$ and Cioce $\mathrm{M}$ : Inhibition of the colony-stimulating-factor-1 receptor affects the resistance of lung cancer cells to cisplatin. Oncotarget 7: 56408-56421, 2016.

11. Gower A, Wang Y and Giaccone G: Oncogenic drivers, targeted therapies, and acquired resistance in non-small-cell lung cancer. J Mol Med (Berl) 92: 697-707, 2014.

12. Kachalaki S, Ebrahimi M, Mohamed Khosroshahi L, Mohammadinejad S and Baradaran B: Cancer chemoresistance; biochemical and molecular aspects: a brief overview. Eur J Pharm Sci 89: 20-30, 2016.

13. Chen WX, Liu XM, Lv MM, Chen L, Zhao JH, Zhong SL, Ji MH, $\mathrm{Hu} \mathrm{Q}$, Luo Z, Wu JZ, et al: Exosomes from drug-resistant breast cancer cells transmit chemoresistance by a horizontal transfer of microRNAs. PLoS One 9: e95240, 2014.

14. Holohan C, Van Schaeybroeck S, Longley DB and Johnston PG: Cancer drug resistance: An evolving paradigm. Nat Rev Cancer 13: 714-726, 2013

15. Ardizzoni A, Boni L, Tiseo M, Fossella FV, Schiller JH, Paesmans M, Radosavljevic D, Paccagnella A, Zatloukal P, Mazzanti P, et al; CISCA (Cisplatin vs. Carboplatin) Metaanalysis Group: Cisplatin- vs. carboplatin-based chemotherapy in first-line treatment of advanced non-small-cell lung cancer: An individual patient data meta-analysis. J Natl Cancer Inst 99: $847-857,2007$.
16. Torigoe $\mathrm{T}$, Izumi $\mathrm{H}$, Ishiguchi $\mathrm{H}$, Yoshida $\mathrm{Y}$, Tanabe $\mathrm{M}$, Yoshida T, Igarashi T, Niina I, Wakasugi T, Imaizumi T, et al: Cisplatin resistance and transcription factors. Curr Med Chem Anticancer Agents 5: 15-27, 2005.

17. Mo EP, Zhang RR, Xu J, Zhang H, Wang XX, Tan QT, Liu FL, Jiang RW and Cai SH: Calotropin from Asclepias curasavica induces cell cycle arrest and apoptosis in cisplatin-resistant lung cancer cells. Biochem Biophys Res Commun 478: 710-715, 2016.

18. Florea AM and Büsselberg D: Cisplatin as an anti-tumor drug: Cellular mechanisms of activity, drug resistance and induced side effects. Cancers (Basel) 3: 1351-1371, 2011.

19. Xie Y, Ma X, Gu L, Li H, Chen L, Li X, Gao Y, Fan Y, Zhang Y, Yao Y, et al: Prognostic and clinicopathological significance of survivin expression in renal cell carcinoma: A systematic review and meta-analysis. Sci Rep 6: 29794, 2016.

20. Zeng LF, Zhang RY, Yu ZH, Li S, Wu L, Gunawan AM, Lane BS, Mali RS, Li X, Chan RJ, et al: Therapeutic potential of targeting the oncogenic SHP2 phosphatase. J Med Chem 57: 6594-6609, 2014

21. Ren Y, Chen Z, Chen L, Fang B, Win-Piazza H, Haura E, Koomen JM and Wu J: Critical role of Shp2 in tumor growth involving regulation of c-Myc. Genes Cancer 1: 994-1007, 2010.

22. Chan G, Kalaitzidis D and Neel BG: The tyrosine phosphatase Shp2 (PTPN11) in cancer. Cancer Metastasis Rev 27: 179-192, 2008.

23. Yang X, Dutta U and Shaw LM: SHP2 mediates the localized activation of Fyn downstream of the $\alpha 6 \beta 4$ integrin to promote carcinoma invasion. Mol Cell Biol 30: 5306-5317, 2010.

24. Cheng SQ, Fan HY, Xu X, Gao WW, Lv SG, Ye MH, Wu MJ, Shen XL, Cheng ZJ, Zhu XG and Zhang Y: Over-expression of LRIG1 suppresses biological function of pituitary adenoma via attenuation of PI3K/AKT and Ras/Raf/ERK pathways in vivo and in vitro. JJ Huazhong Univ Sci Technolog Med Sci 36: 558-563, 2016.

25. Malumbres $M$ and Barbacid M: RAS oncogenes: The first 30 years. Nat Rev Cancer 3: 459-465, 2003.

26. Giltnane JM and Balko JM: Rationale for targeting the Ras/MAPK pathway in triple-negative breast cancer. Discov Med 17: 275-283, 2014.

27. Ksionda O, Melton AA, Bache J, Tenhagen M, Bakker J, Harvey R, Winter SS, Rubio I and Roose JP: RasGRP1 overexpression in T-ALL increases basal nucleotide exchange on Ras rendering the Ras/PI3K/Akt pathway responsive to protumorigenic cytokines. Oncogene 35: 3658-3668, 2016.

28. Zhou XD and Agazie YM: Inhibition of SHP2 leads to mesenchymal to epithelial transition in breast cancer cells. Cell Death Differ 15: 988-996, 2008.

29. Tang C, Luo D, Yang H, Wang Q, Zhang R, Liu G and Zhou X: Expression of SHP2 and related markers in non-small cell lung cancer: a tissue microarray study of 80 cases. Appl Immunohistochem Mol Morphol 21: 386-394, 2013.

30. Li S, Shi H, Ji F, Wang B, Feng Q, Feng X, Jia Z, Zhao Q and Qian G: The human lung cancer drug resistance-related gene BC006151 regulates chemosensitivity in H446/CDDP cells. Biol Pharm Bull 33: 1285-1290, 2010.

31. Tang C, Zhou X, Yang H, Wang Q and Zhang R: Expression and its clinical significance of SHP2 in non-small cell lung cancer. Zhongguo Fei Ai Za Zhi 13: 98-101, 2010 (in Chinese). 